\title{
Structural Analysis of Exoskeleton Model through Human Synchronization Parameters
}

\author{
Rahul R., Tanishq Philip Alexander, Rinkkhesh V., Vikram H. \\ Tamil Nadu, India. \\ * Corresponding author. Tel.: 9677051960; email: rahulkavi30@gmail.com \\ Manuscript submitted November 12, 2017; accepted January 15, 2018. \\ doi: 10.17706/ijbbb.2018.8.3.172-180
}

Sri Venkateswara College of Engineering, Pennalur,Bengaluru High Road, Sriperumbudur, Kancheepuram,

\begin{abstract}
Exoskeleton is a mobile machine integrated with human movements used for applications where it is difficult for humans to handle. Exoskeletons alongside humans breach the revolutionary gap boundary between them. This research paper deals with design solutions for aiding limb module of a powered limb exoskeleton used for handling heavy work load in work-place. An optimal design approach is applied for exoskeletons based on correlations between exoskeleton and limbs in human body. This paper focuses on the various issues with human-centered approach and addressing the problems of physical human-exoskeleton interactions and dealing with everyday scenarios. Initially, a conceptual model was designed and then it was updated to the final design output. Appropriate material selection was done and production of the suit alongside assembly of pneumatic actuators was done. Test analysis was done to check for overall efficiency and conclusion was derived. This paper emphasis on framing the various aspects of design phases in an exoskeleton and provides possible usage of exoskeletons in everyday scenarios. The basic idea behind this research is to conclude on the importance of exo-suits for the future needs.
\end{abstract}

Key words: Exosuits, robots, actuators, stress-strain, humans.

\section{Introduction}

Exoskeleton robots have prospective applications in the field of rehabilitation and patient assistance. They can help weakened and paralysed patients regaining independent life by rehabilitate their mobility and ability to perform activities of daily living (ADL). The primary purpose of assistive technology is for the user to gain independence and self-esteem. An exoskeleton arm is an external mechanical structure which has joints that correlates to the human arms. This integration helps in the transfer of mechanical power to the biological upper-limb [1]. Designing assistive exoskeletons, however, is challenging and complicated as the human factors plays a key role. It also has an important to note that traditional exoskeletons consist of three mutually intersecting orthogonal joints which replicate the spherical motion of the shoulder joint, being the fundamental motion required to create a large workspace according to the activities of daily living (ADL) [2]. All motions in an exoskeleton are examined and controlled by the graphics user interface (GUI). Current upper-limb exoskeleton designs are mainly built for haptic, tele-operations, rehabilitation and strength improvements applications. Tele-operation is the process by which a slave robot is controlled, at a distance, via the replication of forces and movements performed by an operator with the help of an exoskeleton arm [3]. Haptic interface is the interaction of the exoskeleton and the operator through human touch which can 
be used in controlling virtual reality environments [4]. Strength improvement is implemented in exoskeleton devices to aid individuals in bearing or carrying large loads [5].

Previous studies have demonstrated that humans prefer to keep their joints and limbs level mechanics to remain constant when wearing assistive or resistive devices [6], [7]. Along with this invention there are also assistance technology that is evolved. They are: Plantar flexion assisting exoskeletons can reduce the metabolic energy cost of walking improve performance, increase walking speed [8] or restore function in impaired subjects [9]; The ATLAS lower limb active orthoses [10] is another device recently developed by our group intended to be worn by children. These powered devices are intended to enable individuals with certain extremity weaknesses, or who have been affected by SCI to stand up and walk; Recently, haptic interfaces, exoskeletons and robots have been developed to promote self-rehabilitation, arm and wrist rehabilitation [3], [11].

\section{Literature Review}

Initial exoskeleton experiments are commonly done using inexpensive and easy to mold materials aluminum and steel. However, steel is heavy, and the powered exoskeleton must work harder to overcome its own weight to assist the wearer, reducing efficiency. The aluminium alloys used are lightweight, but fail through fatigue quickly; it would be improper for the exoskeleton to fail catastrophically in a high-load condition by "folding up" on itself and cutting the wearer. Soft wearable exoskeletons have been justified to be a valid mean of assisting human movement. Whilst not being compatible for the application of large forces, because of their intrinsic compliance, portability and low-power consumption make them ideal for reducing human muscle effort in activities of daily living such as walking [12] and grasping [13]. A single robot arm has four actuators which lead to increase in the complexity and heaviness of the entire system [14].Soft exoskeletons combine the use of fabrics and a bowden cable-driven actuation for motion transmission to directly induce or apply torques at the joints level. Being flexible and compliant, this kind of systems allow to relocate the actuation unit away from human articulations, and the assistive torque can be transmitted via cables from the actuators to the end effectors [15]. Exoskeletons can be classified into three categories, which allow humans [16]: assist individuals with their daily routine activities; support mobility; and aid therapy by providing mobility.

A flexible sensor technique is developed to measure the pHRI pressure, where the sensory system is composed of several optical-electronic sensors [17]. A flexible pHRI measurement device is designed and applied in the robotic exoskeleton control, which consist of two gasbags and one force sensor connected to each gasbag. A pHRI-based human motion intent estimation is a crucial step for the exoskeleton control. It is essential to get an accurate measurement of the pHRI for the robotic exoskeleton control and the assessment of the assistance grade. The human hand is a sophisticated instrument used to perform many activities of daily living. The opposable thumb is a remarkable anatomical feature of the hand which greatly increases the hand's versatility. Thumb opposition involves flexion, abduction, and medial rotation so that the pulp surface can contact the other digits [18]. The sensory arm master (SAM) exoskeleton [19] has been designed to eliminate singularities in the sagittal plane, while restricted in the transversal and coronal plane. The Intelli Arm is an 8 p 2DOFexoskeleton system for rehabilitation purposes [20], but consists of a mechanical construction that prevents the ease of transportation. In recent studies focussing on neuro rehabilitation, most researchers targeted special therapy to recover the sensory motor function and improve movement coordination in patients with lesions of the central or peripheral nervous system, e.g. after stroke [21]-[24].

Rehabilitation robots can be categorized into three types. First is the sitting/lying type, which is used in the early stage by bedridden patients [19]. Second is the standing/walking type, which is used in the middle 
stage by hospital patients and usually incorporated with a partial body weight support PBWS system, such as Lokomat [25], Reo Ambulatory (Motorika, USA), and ALEX. The application of exoskeletons and active orthoses for human lower limbs has represented a major transformation and improvement in terms of mobility, social interaction and self-image to those who suffer from Spinal Cord Injuries (SCI) or other walking disabilities [26]. Therefore, a variety of commercial devices have hit the market, such as REX, ReWalk and Ekso Bionics. Recently, haptic interfaces, exoskeletons and robots have been developed to promote self-rehabilitation arm and wrist rehabilitation [3], [11]. The Berkeley Lower Extremity Exoskeleton BLEEX was designed to assist people to carry heavy loads, which could walk at the speed of 0.9 $\mathrm{ms}^{-1}$ while carrying $34 \mathrm{~kg}$ pay load [27].

\section{Background Study}

\subsection{Subjects}

When it comes to carrying a backpack, small people can more than hold their own. According to one physicist, a 110-pound adult can tote the heaviest backpack, weighing around 50 pounds. The average human can lift things that weigh much more than their bodyweight if they train to do so. If a normal, otherwise-healthy person with a healthy body composition can't lift at least half their bodyweight they are simply weak and need strength training. The human body has 8 major load-bearing joints. These load-bearing joints are designed to stack vertically and to align horizontally. From the side view, you should be able to pass a plumb line from the earlobe straight down through the ankle.

\subsection{Formulation of Exoskeleton}

Designers need to be aware of design-related commitments made during project planning and project development, as well as proposed mitigation. They also need to be cognizant of the ability to make minor changes to the original concept developed during the planning phase that can result in a "better" final product. The figure given above represents the final output of the exoskeleton for after design changes facilitating ease of use and compatibility.

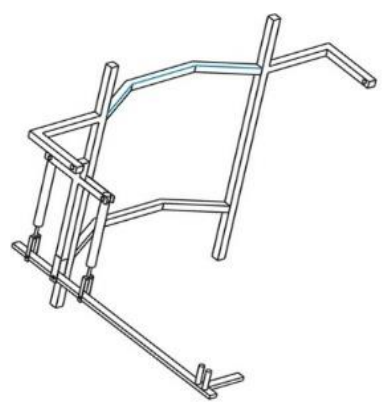

Fig. 1. Conceptual framework model.

\section{System Architecture}

\subsection{Mechanical Structure}

Mild steel is the most commonly used steel. It is used in the industries as well in the different everyday objects we use. The main target of this article is to discuss about different mild steel properties. Almost $90 \%$ steel products of the world are made up of mild steel because it is the cheapest form of steel. Mild steel is not readily tempered or hardened but possesses enough strength.

\subsection{Limb Frames}


Limb Frame is a supportive structure consisting of a T-linkage bar attached to the Main Frame using Rod End Bearing. It holds the Pneumatic Cylinders that attached using U-Clamp and joints. The upper limb has certain structures that support its structure along with its subsystems. These subsystems also ensure the proper placement of muscles and connections that enable co-ordination between those systems. Motions that occur in the upper limb is generally defined in anatomical planes. The basic changes that happen in this system are of two types. Muscle extension for holding the required object and muscle contraction for handling of the weight in the required direction. There are certain supports that the upper limb must have at specific areas in the system. These structures are the ones that are responsible for exerting pressure on the bones or structure for handling loads without any damage to the limb. If supports are not held properly within the system, then each of the subsystems will start failing one by one and ultimately the entire system will collapse. The way or path of allowed motion of the upper limb can be best described by using the concept of degrees of freedom for the parts that form the system of the upper limb. There are three DOF for shoulder joint with the elbow joint having two DOF and the wrist comprising of two DOF. Degrees of freedom stand for the movement allowance for the object or part of consideration. Hence keeping these movement allowances in mind, structures must be designed in such a way to ensure and endure motion through the controlled specified paths during weight handling. Proper care should be taken for the human machine interaction. The motion of the machine part should not put the limb in a dangerous position. This might result in severe damage to the limb. In this way, the support structures were designed for the upper limb keeping all dependent factors in mind.

\subsection{Synthetic Fibre Rig}

Synthetic fibre is man-made fibres, most of them are prepared from raw material (petroleum) called petrochemicals. All fabrics are obtained from fibres and fibres are obtained from artificial or man-made sources. It consists of small unit or a polymer which is made from many repeating units known as monomers. Example: rayon, nylon, polyester, etc. Face arresting A Class was designed to properly mesh the mainframe clamp to the belt to hold the exoskeleton to the body. A buckle with variable size measurement is given for people of varied sizes.

\section{Device Specification}

\subsection{Pneumatic Cylinder}

Pneumatic actuators use pressurised gas to generate output forces. Because of their lightness, pneumatic actuators have a high power to weight ratio. In addition, pneumatic actuators provide a clean and non-flammable actuation option. Air cylinders are measured by three main values: pressure rating, bore and stroke. The principle of physics dictate that the force a cylinder is theoretically capable of producing is identified by multiplying the area of the piston by the air pressure available. Factors such as seal friction, flow coefficients of tubing, fittings and valves, and acceleration and velocity all play a part in reduction of force to one degree or another. The speed at which a cylinder travels is defined by the difference in forces on each side of the piston. An air compressor is used to provide pressurized air. By one of several methods, an air compressor forces more and more air into a storage tank, increasing the pressure. Two pneumatic cylinders are used for load lifting having the following specifications:

Table 1. Pneumatic Cylinder Specification

\begin{tabular}{|c|c|}
\hline Bore & $40 \mathrm{~mm}$ \\
\hline Stroke Length & $100 \mathrm{~mm}$ \\
\hline Pressure & 20 psi - 160 psi (1.3bar to 11 bar) \\
\hline
\end{tabular}




\section{Functional Design Testing}

The purpose of the exoskeleton is to help people compensating the weight of their arms and in-hand objects. The shoulder joint and the flexion of the elbow joint. The exoskeleton is designed with five degrees of freedom, the extra DOF allowing the separation between two shoulder joints, namely, the human and exoskeleton shoulder joints. As shown in Fig. 1, the exoskeleton is mounted on a support bracket, which is worn by a person through a belt and jacket on the trunk. The exoskeleton holds the human arm by grabbing the wrist, with binding between the lower human arm and the lower link. With the captured human arm motion of picking up a cup and drinking, the solved exoskeleton kinematics are shown Fig. 4. In physics, the degree of freedom (DOF) of a mechanical system is the number of independent parameters that define its configuration. Total D.O.F of Exoskeleton is THREE.
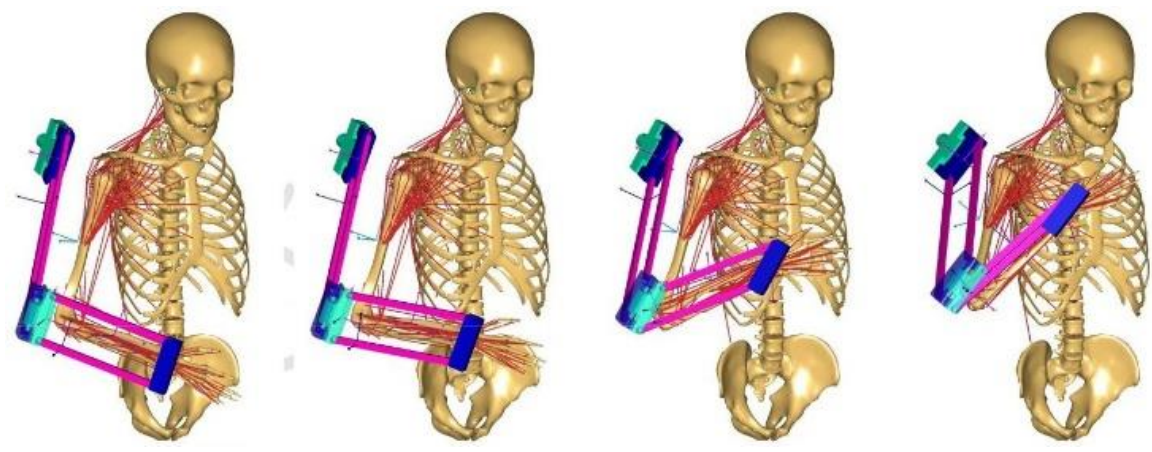

Fig. 2. Human skeletal kinematics

\subsection{Bending Moment}

The need for performing stress analysis is proven from the fact that the structure of the materials changes when it is subjected to forces. The last step in any component design is the process of analyzing for the ability of the device to work under the loading conditions. Analysis done in this manner can generate certain required results which would have been neglected in the analytical method of analyzing the working condition. The analyzing software also takes various parameters into account for the generation of results according to the data given by the user. This result generation is quick and increases productivity while saving time. So, the following observations relating to the object were made.

Bending is the process by which the beam structure changes in such a way that one portion of the beam undergoes compression and the opposite portion of the beam undergoes tension. In our study of Bending Stress on the exoskeleton model, we loaded the arm in steps and found out the stress induced due to bending. At each load step the bending stress was found out. A graph was plotted taking the Load and Bending Stress into account. From the graph it can be inferred that the Bending Stress varies directly with the Load. Both were found to be directly proportional to each other. Shear Force and Bending moment analysis was done, and the results are obtained as follows:

Table 2. Bending Stress

\begin{tabular}{cc}
\hline Load & Bending Stress $\mathbf{~ k g} / \mathbf{c m}^{2}$ \\
\hline 0.5 & 11.27 \\
\hline 1 & 23.07 \\
\hline 2 & 46.15 \\
\hline 3 & 92.30 \\
\hline 6 & 138.46 \\
\hline 8 & 230.77 \\
\hline \hline
\end{tabular}




\subsection{Angular Contraction Observation}

The movement around a particularly localized space can be defined as the range of motion of the object. Range is the concept which indicates the constraint of the object's motion. This also indicates the working space of the object. Through this understanding we can conclude on the path of travel of the object and find out if the object has any hindrance during its operation or if it satisfies the required purpose. In the study made by us on our exoskeleton model, we found out that by loading the arm in steps, the angles of contraction of the arm changed which each loaded step. The initial angle before the start of the operation is 150 degrees. From this point the operation was started and the testing was carried out for the Contraction Angle of the arm. The results are summarized in Table 3.

\begin{tabular}{cc}
\multicolumn{2}{c}{ Table 3. Angle for Load Applied } \\
\hline Load & Angle in Degrees \\
\hline 0.5 & 45 \\
\hline 1 & 45 \\
\hline 3 & 45 \\
\hline 4 & 45 \\
\hline 5 & 45 \\
\hline 6 & 45 \\
\hline 7 & 72 \\
\hline 8 & 120 \\
\hline \hline
\end{tabular}

\subsection{Von Misses Stress Analysis}

The analysis of the component was done by designing the product in a design software, which was followed by application of constraints and load. The software used by us for designing analyzing and simulating the model was CATIA. The model was designed with its constraints were applied. Then the necessary load data was given to the corresponding points after completion of meshing the object. Finally, simulation was done and the results of the analysis for Von Mises Stress were found out as shown in the figure. The maximum stress was found to act near the pivot point of the arm.

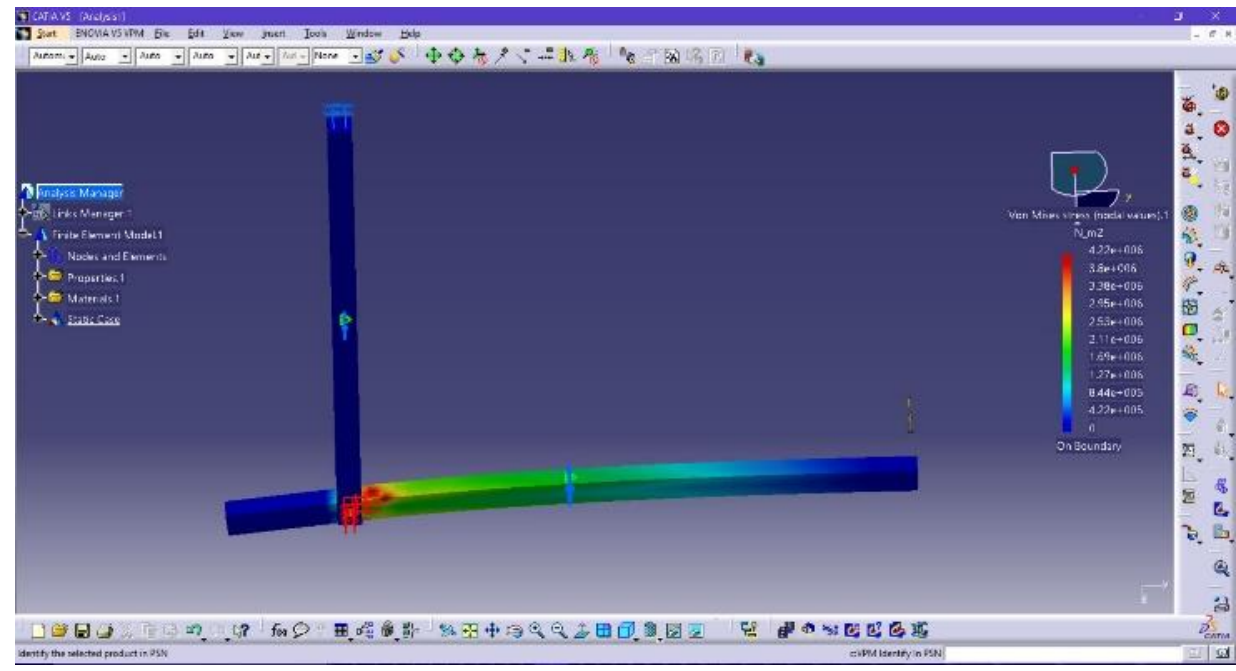

Fig. 3. Von misses stress. 


\section{Conclusion}

The proposed exoskeleton model is demonstrated as a human centered approach involving careful consideration of human joint motions. This model considers the human muscle reactions for careful alignment of the arm with the arm of the human. There is no constraint posed on the human body during the operation of the exoskeleton device. The model proposed here was studied with humans as passive exoskeletons. The observed results indicate the enormous potential and importance of implementation of this exoskeleton into practice in various fields. Because of the device's direct interaction with the human operator, many factors are taken into consideration and the design of the model has been done to avoid placing stress on the human body. The force acted upon during the lifting action is transferred to the back of the human body lowering the risk of snapping joints. Taking all forces and human capabilities into account the exoskeleton model has been proposed and the required analysis has been done. The observed results were tabulated and noted. Hence the model is feasible to use and interacts with humans well, serving its purpose in a various field. The test conducted on the device proves its ability to handle loads for people with problematic limbs. Therefore, the exoskeleton offers an enormous potential for benefitting the humans in various functions.

\section{References}

[1] Panich, S. (2010). Kinematic analysis of exoskeleton suit for human arm. Journal of Computer Science, 6(11), 1272.

[2] Liszka, M. (2006). Mechanical design of a robotic arm exoskeleton for shoulder rehabilitation (Doctoral dissertation).

[3] Lugo-Villeda, L., Frisoli, A., Sandoval-Gonzalez, O., Padilla, M., Parra-Vega, V., Avizzano, C., Ruffaldi, E., \& Bergamasco, M. (2009). Haptic guidance of light-exoskeleton for arm rehabilitation tasks. Proceedings of the 18th IEEE International Symposium on Robot and Human Interactive Communication (pp. 903-908).

[4] Hayward, V., Astley, O. R., Cruz-Hernandez, M., Grant, D., \& Robles-De-La-Torre, G. (2004). Haptic interfaces and devices. Sensor Review, 24(1), 16-29.

[5] Pratt, J. E., Krupp, B. T., Morse, C. J., \& Collins, S. H. (2004). The RoboKnee: An exoskeleton for enhancing strength and endurance during walking. 2004. Proceedings of 2004 IEEE International Conference on Robotics and Automation, (Vol. 3, pp. 2430-2435). IEEE.

[6] Chang, Y. H., Roiz, R. A., \& Auyang, A. G. (2008). Intralimb compensation strategy depends on the nature of joint perturbation in human hopping. Journal of Biomechanics, 41, 1832-1839.

[7] Kao, P. C., Lewis, C. L., \& Ferris, D. P. (2010). Invariant ankle moment patterns when walking with and without a robotic ankle exoskeleton. Journal of Biomechanics, 43, 203-209.

[8] Norris, J. A., et al. (2007). Effect of augmented plantarflexion power on preferred walking speed and economy in young and older adults. Gait Posture, 25, 620-627.

[9] Takahashi, K. Z., et al. (2015). A neuromechanics-based powered ankle exoskeleton to assist walking post-stroke: a feasibility study. J. Neuroeng. Rehabil., 12, 1-13,

[10] Garcia, E., Sanz-Merodio, D., Sanchez, F., Arevalo, J., \& Gonzalez-de-Santos, P. (2011). Development of the atlas lower-limb active orthosis. International Conference on Climbing and Walking Robots (CLAWAR). Paris, France.

[11] Oblak, J., Cikajlo, I., \& Matjacic, Z. (2010). Universal haptic drive: a robot for arm and wrist rehabilitation. IEEE Transactions on Neural Systems and Rehabilitation Engineering, 18(3), 293-302.

[12] Asbeck, A. T., et al. (2015). Soft exosuit for hip assistance. Robot. Auton. Syst., 73, 102-110.

[13] Xiloyannis, M., et al. (2016). Modelling and design of a synergy-based actuator for a tendon-driven soft 
robotic glove. Proceedings of 6th IEEE International Conference on Biomedical Robotics and Biomechatronics (pp. 1213-1219).

[14] Palli, G., \& Melchiorri, C. (2006). Model and control of tendon-sheath transmission systems. Proceedings of IEEE International Conference on Robotics and Automation (pp. 988-993).

[15] Akdoğan, E., \& Adli, M. A. (2011). The design and control of a therapeutic exercise robot for lower limb rehabilitation: Physiotherabot. Mechatronics, 21(3), 509-522.

[16] Donati, M., et al. (2013). A flexible sensor technology for the distributed measurement of interaction pressure. Sensors, 13, 1021-1045.

[17] Jones, L. (2006), Human Hand Function. Oxford University Press, Oxford, New York, NY.

[18] Letier, P., et al. (2008). SAM: A 7-DOF portable arm exoskeleton with local joint control. Proceedings of 2008 IEEE/RSJ International Conference on Intelligent Robots and Systems (pp. 3501-3506).

[19] Ren, Y., Park, Y.-S., \& Zhang, L.-Q. (2009). Developing a whole-arm exoskeleton robot with hand opening and closing mechanism for upper limb stroke rehabilitation. Proceedings of IEEE 11th International Conference on Rehabilitation Robotics (pp. 761-765).

[20] Mayhew, D., Bachrach, B., Rymer, W. Z., \& Beer, R. F. (2005). Development of the MACARM - a novel cable robot for upper limb neurorehabilitation. IEEE. pp. 299-302.

[21] Rosati, G., Gallina, P., \& Masiero, S. (2007). Design, implementation and clinical tests of a wire-based robot for neuro rehabilitation. IEEE Transactions on Neural Systems and Rehabilitation Engineering, 15(4), 560-569.

[22] Krebs, H. I., Volpe, B. T., Williams, D., Celestino, J., Charles, S. K., Lynch, D., \& Hogan, N. (2007). Robot-aided neurorehabilitation: a robot for wrist rehabilitation. IEEE Transactions on Neural Systems and Rehabilitation Engineering, 15(3), 327-335.

[23] Ren, Y., Kang, S., Park, H., Wu, Y., \& Zhang, L. (2012). Developing a multi-joint upper limb exoskeleton robot for diagnosis, therapy and outcome evaluation in neurorehabilitation. IEEE Transactions on Neural Systems and Rehabilitation Engineering, 21(3), 490-499.

[24] Li, J., Zheng, R., Zhang, Y., \& Yao, J. (2011). iHandRehab: An interactive hand exoskeleton for active and passive rehabilitation. Proceedings of 2011 IEEE International Conference on Rehabilitation Robotics (ICORR) (pp. 1-6).

[25] Jezernik S., Colombo G., Keller T., Frueh H., \& Morari, M. (2003). Robotic Orthosis Lokomat: A rehabilitation and research tool. Neuromodulation, 6(2), 108-115.

[26] Cestari, M., Sanz-Merodio, D., Arevalo, J., \& Garcia, E. (2012). Development of synergic multiarticular actuation in a lower-limb active orthosis. Proceedings of 13th Mechatronics Forum International Conference, Linz, Austria.

[27] Kazerooni, H., Steger, R., \& Huang, L. (2006). Hybrid control of the berkeley lower extremity exoskeleton (BLEEX). International Journal of Robotics Research, 25, 561-573.

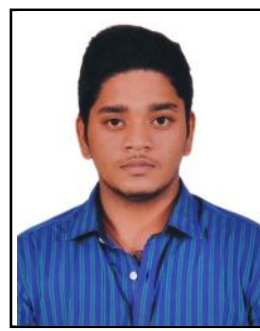

Rahul Rajan is currently pursuing his final year in Sri Venkateswara College of Engineering. His interests are involved in society improvements, which made him invest his knowledge in development of such similar territories. He is very much passionate about becoming a socio-entrepreneur \& shift the economic resources out of his country and soon make it spread globally. 


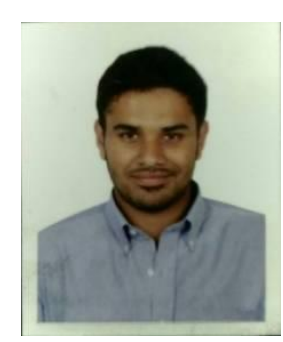

Tanishq Philip Alexander is pursuing his final year in Sri Venkateswara College of Engineering. His interests lie in design and society development and improvement. He is an engineer who's interests are in the different areas that isn't usually sought after, a mix of field and desk work.

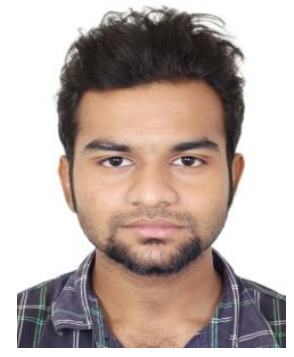

Rinkkhesh V. is doing his final year mechanical engineering course in Sri Venkateswara College of Engineering. Right from his childhood he has been fascinated about various objects and devices used in daily life. His curiosity in this field grew more with the astounding technological improvements during the years. This led him to take science as his main stream during his high schooling. Due to his research interest he has presented a paper in a International and National Conference. He has also got the best paper award for both. Hence his interest drives him to do many things apart from his regular curriculum. His spirit of learning will continue forever.

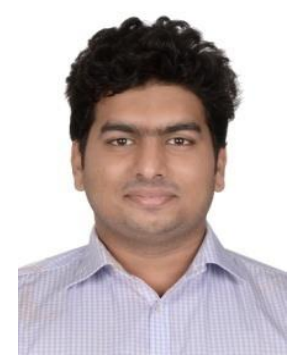

Vikram H. is a graduate student from Chennai pursuing a bachelor degree in mechanical engineering at Sri Venkateswara College of Engineering Batch 2014-18. His areas of interests are industrial engineering, supply chain, and production management, as well as manufacturing studies. He has done his internship at FORD INDIA Pvt. Limited and Amalgamation Repco - Chennai. Mr. Vikram H has attended various conferences and published papers in well renowned journals. He also holds the post of General Secretary - Quality Management Center, SVCE. 